\title{
In memoriam Otto Martin Hess
}

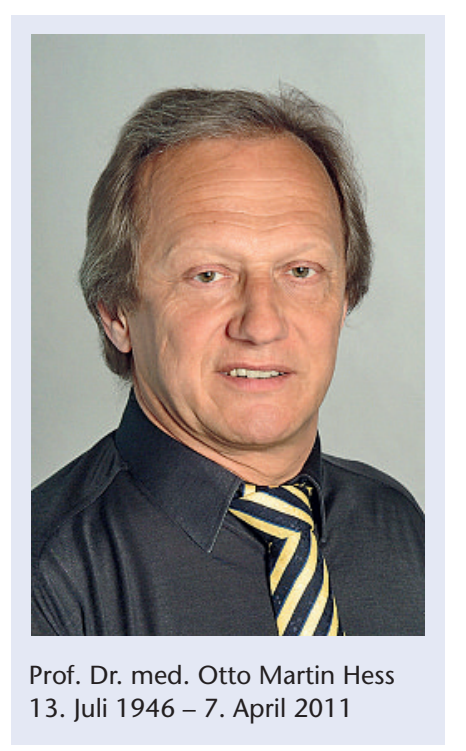

Otto Hess hat uns verlassen, und wir können es noch nicht fassen. Komplikationen eines lebensbegleitenden chronischen Leidens haben ihn aus voller beruflicher Tätigkeit und erfülltem sozialem Leben herausgerissen und seinem Dasein zur Bestürzung aller innert Wochen ein Ende gesetzt. Sein 65. Geburtstag und damit seine Pensionierung standen unmittelbar bevor. Er sah sie nicht als einen Zwischenhalt, sondern allenfalls als eine Boje, an der man vorbeisegelt, ohne wirklich die Richtung zu ändern oder Wind aus den Segeln zu nehmen. Er hat die Boje nicht erreicht.

Otto Hess wurde in Illnau-Effretikon geboren und blieb der Gegend zeitlebens treu. Er besuchte das Gymnasium in Winterthur und studierte Medizin an der Universität Zürich, wo er 1973 promovierte. Wilhelm Rutishauser, der damalige Leiter der jungen kardiologischen Abteilung der medizinischen Poliklinik erkannte sein Talent bei der mündlichen Staatsexamensprüfung, engagierte ihn auf der Stelle und legte den Grundstein für einen hervorragenden Kardiologen, den wir nun verloren haben.

Die zahlreichen Talente, die hohe Intelligenz, der unstillbare Wissensdurst, die initiative, effiziente und zuverlässige Art, Aufträge zu erledigen, und das angenehme Wesen Ottos nahmen auch seine weiteren Vorgesetzten für ihn ein. Hans Peter Krayenbühl übernahm die Rolle des direkten Tutors von Otto Hess nach der Wegberufung von Wilhelm Rutishau- ser nach Genf. Walter Siegenthaler hätte Otto gerne aus der Abteilung für Kardiologie in die Allgemeine Innere Medizin transferiert und machte Otto zum Privatassistenten, um ihm diesen Wechsel schmackhaft zu machen. Er musste indes erkennen, dass Ottos Herz dem Herzen gehörte. Bis zu seiner Emeritierung hielt Walter Siegenthaler grosse Stücke auf Otto Hess, obwohl ihm dieser sein Angebot, ihn als persönlichen Mitarbeiter zu fördern, ausgeschlagen und ihm nicht nur in fachlichen Diskussionen immer wieder Paroli geboten hatte. 1980 brach Otto frisch verheiratet mit der Organistin Elvira in Begleitung des 1979 geborenen Töchterchens Simone für einen mehrjährigen Weiterbildungsaufenthalt nach San Diego, Kalifornien, auf. Er wollte vom dortigen kardiovaskulären Pathophysiologie-Papst John Ross ergänzend lernen, was die diesbezüglich ebenfalls Weltruf geniessende Zürcher Schule nicht zu bieten hatte. Es ging auch darum, seine bereits erfolgreich angelaufene Karriere als Akademiker und Fachliteraturautor zu intensivieren. Der Aufenthalt blieb kürzer als geplant, da eine Oberarztstelle auf der Kardiologie der medizinischen Poliklinik am Universitätsspital in Zürich frei wurde und man nichts unterliess, Otto für diese Stelle frühzeitig zurückzuholen.

1983 wurde er zum Privatdozenten der Inneren Medizin, speziell Kardiologie, und 1989 wurden ihm gleich zwei Medizinalprofessuren verliehen, die Titularprofessur der Universität Zürich und eine lebenslange Gastprofessur der Universität Bari, Italien. Andere Gastprofessuren folgten.

*

Zahlreiche hochkarätige Preise pflasterten seinen akademischen Weg. 1982 war er einer der ersten Preisträger der Schweizerischen Stiftung für Kardiologie, die damit seine Habilitationsschrift «Diastolische Funktion des linken Ventrikels» auszeichnete. 1986 kam der Fränkel-Preis der Deutschen Gesellschaft für Herzund Kreislaufforschung dazu, 1987 der 1. Preis der Schweizerischen Gesellschaft für Innere Medizin, 1991 der Götz-Preis der Universität Zürich, 1996 der Franz Loogen-Preis der Universität Essen, Deutschland, und 2007 der Paul Morawitz Forschungspreis der Deutschen Gesellschaft für Kardiologie.

Otto Hess gründete die Arbeitsgruppe für Herzinsuffizienz der Schweizerischen Gesellschaft für Kardiologie und war massgeblich beteiligt an der Formierung der entsprechenden Arbeitsgruppe der Europäischen Gesellschaft für Kardiologie. Er organisierte zahllose Veranstaltungen mit allgemeinen kardiologi- 
schen Inhalten und über Spezialthemen wie Herzinsuffizienz, Pathophysiologie und neue Bildgebung. Er war gesuchter Redner und Vorsitzender an Jahrestagungen schweizerischer, europäischer und aussereuropäischer Fachgesellschaften und arbeitete im Herausgeberrat namhafter internationaler Zeitschriften mit. Die Liste seiner Publikationen als Erst- und Letztautor ist lang. Noch nachhaltiger war seine Tätigkeit als Motivator für Studenten, jüngere Kollegen, Altersgenossen und auch Vorgesetzte, wenn es darum ging, Studien zu planen, durchzuführen, auszuwerten und zu publizieren, aber auch wenn es darum ging, aus publizierten Arbeiten das Wesentliche und Nützliche herauszukristallisieren.

1996 wurde Otto Hess als Nachfolger des als Leiter der Kardiologie ans Universitätsspital Zürich berufenen Thomas Lüscher zu meinem Vertreter als Chefarzt der Kardiologie der Universität Bern ans Inselspital berufen. In den knapp 15 Jahren, die er in Bern wirkte, hat er viel bewegt, mit Verve Studenten unterrichtet, jungen Kolleginnen und Kollegen als Steigbügelhalter aufs berufliche Pferd geholfen, um ihnen dann gleichsam vorauszugaloppieren, sei es nun klinisch oder akademisch. All dies hat seinen nationalen und internationalen Ruf als kardiologische Koryphäe und Meinungsbildner in verschiedenen Subspezialitäten gefestigt.

Während sechs Jahren, bis kurz vor seinem Tod, hat er eines der umsatzstärksten Departemente des Inselspitals geleitet, das Departement Herz und Gefässe. Über sein Geschick hinaus, mit Nichtärzten über medizinische Themen zu diskutieren, hat er sich dabei auch lokalpolitisch ausgezeichnet in einer Zeit, in der die Universitätsklinik vom gemeinnützigen hochspezialisierten Dienstleister und geisteswissenschaftlichen Institut zum kostenorientierten Wirtschaftsunternehmen mutierte.
Kann man vielseitiger sein, als Otto es war? Er war Schöngeist und liebte Literatur, Musik, Kunstmalerei, Bildhauerei und Veteranenfahrzeuge mit grossem Sachverständnis. Er war Gourmet und Weinkenner. Er war passiver und aktiver Sportaficionado. Sein letzter Einsatz in einem Fussball-Grümpelturnier liegt kein Jahr zurück. Er war sozial eng vernetzt, in seinem beruflichen wie auch privaten Umfeld. Er war ein einfühlsamer und erfolgreicher Doktor seiner Patienten, denen er nicht nur sein Wissen und handwerkliches Können im Herzkatheterlabor angedeihen liess, sondern auch Mitgefühl, Verständnis, aber eben auch mal gesunde Kritik. Otto war Erfinder, vor allem in seiner Berner Zeit. Neben der kultverdächtigen Multivitamin-Präventionspille hat er Koronarstents entwickelt und Prototypen von künstlichen Herzklappen, die nun leider ohne ihn zur Marktreife gebracht werden müssen.

Seine erste Tochter Simone ist Juristin geworden. Ihr ist er ein bleibendes Vorbild bezüglich Energie, Schaffenskraft, positive Weltanschauung und menschlicher Wärme. Seine zweite Tochter Nina wird bald Ärztin sein und ihn zusätzlich in dieser Beziehung als Rollenmodell haben. Wir am Inselspital werden ihn auf Schritt und Tritt vermissen. Seine Fach- und Lehrmeinungen werden nachhallen, seine zahlreichen Publikationen wie Originalartikel, Übersichtsarbeiten, Buchkapitel und ganzen Bücher werden noch lange konsultiert werden. Das geflügelte Wort «Nicht verzagen, Otto fragen» wird in der Inselspital-Kardiologie fortklingen, aber leider nicht mehr umsetzbar sein.

Prof. Dr. med. Bernhard Meier, Inselspital Bern 